\title{
Dix propositions sur les valeurs
}

10 Proposals on Values

\section{Nathalie Heinich}

\section{CpenEdition}

\section{Journals}

Édition électronique

URL : https://journals.openedition.org/questionsdecommunication/11156

DOI : 10.4000/questionsdecommunication. 11156

ISSN : 2259-8901

\section{Éditeur}

Presses universitaires de Lorraine

\section{Édition imprimée}

Date de publication : 1 septembre 2017

Pagination : 291-313

ISBN : 9782814303256

ISSN : 1633-5961

\section{Référence électronique}

Nathalie Heinich, «Dix propositions sur les valeurs », Questions de communication [En ligne], 31 | 2017, mis en ligne le 01 septembre 2019, consulté le 26 octobre 2021. URL : http://journals.openedition.org/ questionsdecommunication/11156; DOI : https://doi.org/10.4000/questionsdecommunication. 11156 


\section{$>$ ÉCHANGES}

NATHALIE HEINICH

Centre de recherches sur les arts et le langage École des hautes études en sciences sociales Centre national de la recherche scientifique

F-75006

heinich@ehess.fr

\section{PROPOSITIONS SUR LES VALEURS}

Résumé. - L'article propose un condensé en dix points de l'ouvrage Des valeurs. Une approche sociologique (Gallimard, 20 I7), dont ont été extraits les passages les plus susceptibles d'entraîner des discussions voire des controverses. Y sont notamment ciblées les réductions de la notion de valeur opérées par l'économie classique et néo-classique, par la sociologie quantitative des valeurs, et par la plupart des approches philosophiques de cette notion.

Mots clés. - évaluation, philosophie des valeurs, pragmatique des valeurs, sociologie des valeurs, valeur, valeurs, valeur économique 
$\mathrm{I}$

e propose ci-dessous dix extraits de mon livre Des valeurs. Une approche sociologique (Gallimard, 2017), choisis pour susciter le débat en raison de leur caractère contreintuitif, paradoxal voire sciemment polémique.

Ils concernent, premièrement, la mise à mal de quelques idées reçues sur la notion de valeur : sa réduction à l'évaluation chiffrée ; sa réduction au résultat des évaluations, « la » valeur, au détriment des deux autres dimensions que sont les biens et les principes ; la réduction « des » valeurs aux valeurs de la droite ; leur assimilation à des entités religieuses ou « sacrées »; leur réduction à des intérêts cachés ; leur invalidation par manque d'universalité, ou par manque de réalité.

Le deuxième extrait propose une définition de la notion de valeur, comme étant « la résultante de l'ensemble des opérations par lesquelles une qualité est affectée à un objet ». Cette définition permet une approche pragmatique, centrée sur les opérations d'évaluation : opérations qui sont fonction à la fois de la nature de l'objet évalué, de la nature des sujets évaluateurs et de la nature du contexte d'évaluation.

Le troisième extrait met en évidence les trois sens du mot valeur - la grandeur, le bien, le principe - et en développe les implications, notamment méthodologiques.

Le quatrième extrait traite des quatre catégories d'objets susceptibles d'appeler une évaluation (soit par l'attachement, soit par la mesure, soit par le jugement) et, par conséquent, d'être crédités d'une valeur : à savoir les choses, les personnes, les actions, les états du monde. Ainsi s'agit-il d'éviter les apories entraînées par la réduction de la problématique de la valeur à la seule valeur des choses, dans la voie ouverte par la science économique et trop souvent suivie par la sociologie, à l'exclusion des trois autres.

Le cinquième extrait se concentre sur le troisième sens du mot valeur, les principes d'évaluation, et propose quelques critères permettant de les identifier. II s'agit ici de s'extraire de la réduction de la problématique des valeurs au deuxième sens du terme - les biens - qui affecte trop souvent les enquêtes quantitatives en sociologie des valeurs.

Le sixième extrait plaide pour une approche pragmatiste des valeurs, à travers l'étude empirique des processus d'évaluation, seule à même de rendre compte de leur complexité et de produire une analyse sociologique qui soit tout à la fois neutre, descriptive, empirique et compréhensive.

Le septième extrait explicite le tournant « grammatical » qui, dans le sillage ouvert par les inflexions du pragmatisme dans la sociologie française de la dernière génération, permet de mettre en œuvre une approche qualitative, pluraliste et compréhensive.

Le huitième extrait situe l'approche adoptée par rapport aux trois grandes options théoriques que sont le structuralisme, l'interactionnisme et le constructivisme en plaidant pour un usage non exclusif mais, au contraire, perspectiviste, adapté aux différentes facettes de l'objet de recherche. 
Le neuvième extrait explicite les apories propres à l'alternative stérile entre une conception universaliste (ou objectiviste) et une conception relativiste (ou subjectiviste) des valeurs - alternative qui a beaucoup occupé mais aussi beaucoup stérilisé les conceptions philosophiques de la notion de valeur.

Le dixième extrait propose une approche proprement sociologique de la notion de valeur, permettant de sortir de cette alternative.

J'espère que ces dix propositions permettront d'ouvrir des discussions.

\section{Pour en finir avec quelques idées reçues}

Précisons d'emblée ce qu'il convient de ne pas chercher dans ce livre, car on ne l'y trouvera pas.

Tout d'abord, débarrassons-nous de la focalisation sur le « juste prix 》 des choses: la valeur n'est pas - nous le verrons au premier chapitre - réductible « en dernière instance » à son évaluation chiffrée qu'est le prix. On le voit bien dans le cas de l'art, où les interrogations récurrentes sur « la valeur des œuvres» tendent à occulter et la relativité intrinsèque des évaluations qui en sont faites, et la variété des façons d'attribuer de la valeur, par les mots et par les actes et pas seulement par l'évaluation chiffrée'. La monopolisation du terme de « valeur» par l'économie, et sa réduction corrélative à la question tant de sa mesure par le prix que de la correspondance de celui-ci avec une supposée « valeur » intrinsèque des objets, peut avoir du sens dans une logique utilitaire ; mais s'agissant de comprendre ce qu'est une valeur, elle équivaut à prendre l'arbre pour la forêt.

La valeur est d'autant moins réductible au prix qu'elle ne se limite pas à ce que « vaut » une chose, mais s'étend aussi aux biens auxquels on confère une valeur, ainsi qu'aux principes permettant de conférer cette valeur : « la » valeur s'inscrit donc - nous le verrons en deuxième partie - dans une pluralité d'objets « de » valeur, et résulte d'une pluralité de « valeurs » mobilisées pour en juger. C'est dire qu'en amont de la question de « la » valeur, qui tend à focaliser les débats, il importe de remonter « aux 》 valeurs qui la fondent. Le pluriel « des » valeurs primera, par conséquent, sur le singulier de « la » valeur. Et parmi ces « valeurs » au pluriel, celles qui ressortissent à des principes nous occuperont beaucoup plus que celles, mieux connues, qui ressortissent à des biens, comme on le verra dans la troisième partie.

Une troisième idée reçue dont ce livre devrait nous débarrasser consiste à penser que ces « valeurs » au pluriel - biens ou principes - sont particulièrement en phase avec une position droitière en politique. Certes, le vocabulaire des « valeurs » a été fortement sollicité par les partis de droite dans la France de la dernière génération,

\footnotetext{
' Cf. N. Heinich, « L'évaluation de l'art contemporain », in Bertrand Boquet (éd.), La Fièvre de l'évaluation,
} Lille, Presses universitaires du Septentrion, 2016. 
avec la focalisation sur la famille, la nation, la religion, l'autorité etc. ${ }^{2}$; mais l'acception progressiste, quoique moins facilement abritée derrière le mot de « valeur », est tout aussi présente, s'agissant par exemple de mettre en exergue l'égalité, les droits de l'homme ou - de façon plus complexe aujourd'hui - la laïcité 3 . Bref : les valeurs ne sont ni de droite ni de gauche, et on en trouvera de multiples confirmations dans la « sociologie axiologique » présentée en troisième partie.

Une quatrième idée qui vient perturber la réflexion sur les valeurs consiste à leur attribuer un caractère « sacré », soit pour leur conférer du poids soit, au contraire, pour les discréditer, selon l'usage implicitement négatif de la réduction au religieux qui s'est imposé dans les sciences humaines et sociales. Mais c'est là confondre le partage à grande échelle d'une commune visée avec un dispositif de valorisation par la séparation d'avec le monde ordinaire ; et c'est également supposer que toute transcendance serait forcément religieuse ou, en d'autres termes, que « la religion » aurait le monopole de la transcendance, alors qu'elle n'en est qu'une des formes parmi d'autres : une erreur de raisonnement typique d'une pensée encore modelée dans le moule religieux $x^{4}$. Pour comprendre un objet quelque peu mystérieux, tel que les valeurs, mieux vaut éviter de le réduire à un objet encore moins élucidé, tel que « le sacré », dont l'invocation permet au penseur paresseux de se présenter à peu de frais comme plus malin que le commun de ses lecteurs. Le paradigme religieux est sans pertinence concernant la question des valeurs et - comme souvent - bloque la pensée beaucoup plus qu'elle ne l'ouvre.

Plus trivialement, une conception quelque peu orientée de la sociologie a répandu un autre préjugé : celui consistant à réduire les valeurs à des intérêts cachés. Elles ne seraient donc rien d'autre qu'une « idéologie » utilisée par les « dominants » pour asseoir leurs privilèges sur des principes qu'ils feraient partager aux « dominés ». Nous verrons dans la première partie, en observant les jugements de valeur effectifs tels qu'ils s'expriment concrètement dans leurs contextes (car c'est là,

\footnotetext{
${ }^{2}$ « La notion de valeur semble être toujours liée à celle de tradition. Cela tient sans doute à l'activité déployée par ceux qui, effrayés par le changement social et l'évolution des mentalités, s'attachent à restaurer la force de règles morales dont le pouvoir de contrainte leur parait s'être affaibli. Pour cette pensée conservatrice, arc-boutée sur la grandeur de l'héritage et la nécessité de le transmettre dans sa pureté rêvée, les valeurs sont les piliers de l'ordre du monde qu'il faut scrupuleusement veiller à respecter : autorité, famille, religion, procréation normale, sexualité correcte. Et il est d'autant plus impératif de le faire que cet ordre est présenté comme divin ou naturel. Dans ce raisonnement, bafouer ces valeurs revient ni plus ni moins qu'à (sic) dissoudre la société ou détruire la civilisation » (A. Ogien, « Qu'est-ce qui fait valeur dans notre société? », in B. Bocquet (éd.), La Fièvre de l'évaluation, Lille, Presses universitaires du Septentrion, 2016, p. 23-24).

${ }^{3}$ S'opposant à cette vision passéiste du caractère éternel et immuable des valeurs, les partisans de la modernité se sont résolus à reprendre le mot valeur pour faire entendre, dans le discours public, une voix différente. Pour eux, les valeurs ne proviennent pas d'un fond immémorial qu'il convient de perpétuer à l'identique jusqu'à la fin des temps. Elles sont, au contraire, orientées vers le futur et prônent l'émancipation, l'humanisme, l'égalité, le droit de la personne, la läicité, la République ou la Raison » (ibid., p. 24).

${ }^{4}$ Cf. N. Heinich, « Des limites de l'analogie religieuse : l'exemple de la célébrité », Archives de sciences sociales des religions, $n^{\circ} \mid 58$, avril-juin 2012.
} 
et seulement là, que les valeurs se donnent à voir), à quel point cette politisation simpliste de la question des valeurs est éloignée de la réalité.

Un autre péché mignon de la sociologie critique consiste à réduire les valeurs à leurs ancrages contextuels et, partant, à les invalider par défaut d'universalité : telle valeur n'apparaîtrait « que » dans la société occidentale actuelle, par exemple, et ne pourrait donc pas légitimement prétendre au titre de « valeur ». Or, nous verrons que toutes les valeurs sont contextuelles - comme le sont, d'ailleurs, toutes les formes de l'expérience humaine. Cela n'empêche nullement de les invoquer en tant que visées devant être partagées dans la société à laquelle on appartient. Qu'une représentation ne soit ni une substance ni un fait objectif existant en dehors de l'esprit humain, qu'elle soit donc « socialement construite », voire « mythique », ne lui ôte rien de son effectivité et de son efficacité, parce que la vie commune est faite non seulement de choses matérielles et d'institutions, mais aussi de conceptions plus ou moins partagées, qu'elles s'expriment par des mots, par des chiffres ou par des actes. Parmi ces représentations, les représentations « axiologiques 》, autrement dit les valeurs, ne sont pas les moins répandues ni les moins agissantes. De l'acteur affirmant ses valeurs et du sociologue critique les infirmant au nom de leur manque d'objectivité ou d'universalité, le plus naïf des deux n'est pas toujours celui qu'on croit.

II serait donc tout aussi naïf de s'imaginer que l'on ne peut comprendre ce qu'est une valeur qu'en s'intéressant aux valeurs « en soi ». Car contrairement à ce que voudrait une approche spontanément métaphysique, c'est en s'intéressant à ce que sont les valeurs « pour soi », autrement dit pour les acteurs, que la sociologie peut nous éclairer. Cet ouvrage nous parlera donc de la relation que les acteurs entretiennent avec les valeurs, de l'usage qu'ils font de leurs évaluations. II relève ainsi, pourrait-on dire, d'une « sociologie analytique », analogue à la « philosophie analytique » au sens où celle-ci, selon Arthur Danto, est une enquête non sur le monde, mais sur « les manières dont on pense le monde et dont on en parle $»^{5}$.

Enfin, en nous débarrassant de la tentation de réduire les valeurs à des « faits 》 objectifs - soit pour les valider, soit pour les invalider en en pointant la relativité ou la subjectivité -, nous éviterons l'erreur consistant à croire que l'on peut disqualifier une valeur en démontrant qu'elle « n'existe pas », au sens où elle ne serait pas effectivement mise en œuvre (par exemple, « l'égalité, ça n'existe pas »). Mais c'est confondre une visée de l'action avec ses résultats. Ainsi, s'acharner à prouver que l'égalité dans le monde scolaire est une illusion parce que l'Education Nationale ne parvient pas à assurer l'égalité des positions ni même des chances, c'est constater, certes, une réalité hautement critiquable, mais c'est en même temps s'aveugler sur ce qui permettrait de la faire progresser, en passant à côté de ce fait, fondamental en démocratie, qu'est le partage de la valeur d'égalité, en tant que mission impartie à l'institution scolaire - même si celle-ci la remplit mal. Et c'est donc, du même coup, miner en la disqualifiant la seule ressource permettant d'exiger la bonne mise en œuvre de cette mission. Ainsi, c'est au nom d'une conception de la démocratie

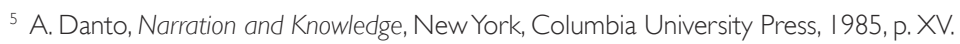


vidée de toute prise au sérieux de la notion de valeurs que certains, croyant œuvrer pour la démocratisation, s'ingénient à lui couper les ailes. Là encore, les plus démystificateurs ne sont pas les moins mystifiés qui soient. Voilà qui devrait inciter plus encore à s'intéresser de près à la question des valeurs.

(« Introduction »)

\section{Définir la valeur}

II serait dommageable de réduire la question de la valeur à celle du prix, familière tant à l'économie qu'aux usages de sens commun. L'on s'interdirait ainsi d'observer et de comprendre les multiples façons dont les acteurs parviennent à coordonner leurs attachements, leurs mesures et leurs jugements, en conférant une évaluation plus ou moins positive à des objets au statut beaucoup plus varié que ne le sont les choses, au nom d'une « grandeur » intrinsèque qu'ils posent comme cause de leurs évaluations alors même que le sociologue n'a accès, empiriquement, qu'à leurs conséquences et à leurs modalités, à travers les processus d'attribution de cette valeur.

Certes, le prix a pour lui d'être directement observable, au contraire de la valeur. Ce n'est pas une raison pour réduire celle-ci à l'état d'illusion, ou de stratégie utilisée par les acteurs pour contester ou justifier un prix, comme le font Luc Boltanski et Arnaud Esquerré dans leur critique de la notion de valeur : quoique n'ayant pas de réalité matérielle hormis ses manifestations concrètes, une représentation collective, discutée, argumentée, voire instituée, n'est pas pour autant une illusion, comme le voudrait l'usage que fait la pensée critique de la notion de « construction sociale », réduisant la notion de représentation mentale, au mieux à un point aveugle des sciences sociales, au pire à une illusion tout juste bonne à balayer'.

Ce cadre étant posé, il devient possible de proposer la définition suivante de la valeur au sens premier du terme : la valeur est la résultante de l'ensemble des opérations par lesquelles une qualité est affectée à un objet, avec des degrés variables de consensualité et de stabilité7. Ces opérations sont fonction à la fois de la nature de l'objet évalué, de la nature des sujets évaluateurs et de la nature du contexte d'évaluation. C'est dire qu'à l'image des opérations qui la produisent, la valeur n'est ni objective, ni subjective, ni arbitraire : elle est motivée par les prises qu'offre l'objet à l'évaluation, par les représentations collectives dont sont dotés les individus, et par les possibilités qu'offrent les contextes d'activation de ces représentations à propos d'un objet.

\footnotetext{
${ }^{6}$ Cf. N. Heinich, « Représentation : la dimension cognitive du terme », Sociétés et représentations, $n^{\circ} 40$, automne 2015 ; Le Bêtisier du sociologue, op. cit.

7 Une définition analogue avait été proposée concernant la notion d'identité : l'identité est la résultante de l'ensemble des opérations par lesquelles un prédicat est affecté à un sujet (cf. N. Heinich, Etats de femme. L'identité féminine dans la fiction occidentale, Paris, Gallimard, 1996).
} 
Ainsi peut se construire une définition proprement sociologique de la valeur, qui permet d'en étudier concrètement, empiriquement, les manifestations, en tant que celles-ci mettent en œuvre des « valeurs » au troisième sens du terme - des valeurs-principes -, créant des « valeurs 》 au deuxième sens du terme - des valeursobjets, autrement dit des « biens ».

(chapitre $7:$ : Valeur-grandeur : ce que valent les choses »)

\section{Les trois sens du mot « valeur»}

La première signification utilise essentiellement l'article défini au singulier (« la valeur »), alors que les deux autres acceptent aussi l'article indéfini (« une valeur ») et le pluriel (« les valeurs », « des valeurs »). Selon le premier sens donc, «la » valeur équivaut à la grandeur intrinsèque d'un objet quelconque, motivant son appréciation positive : en fonction des objets auxquels il s'applique, le terme peut être synonyme d'《 importance », de « mérite », de « qualité », de « quantité », de 《 vertu » (au sens antique de « virtus »), voire de « prix » au sens figuré. Le sens propre de ce dernier terme y a aussi sa place, dans la mesure où cette « valeur » peut souvent (mais pas toujours, nous le verrons) se mesurer, notamment par le prix affecté à l'objet. C'est la signification la plus familière au sens commun, ainsi qu'aux économistes : «Quelle est la valeur de cette montre? ?, autrement dit « Que vaut cette montre? ?, « Quel prix est-on disposé à la payer? ». Nous appellerons ce premier sens du mot valeur la « valeur-grandeur $»^{8}$.

Dans la deuxième signification, « une » valeur est un objet crédité d'une appréciation positive, autrement dit un objet communément considéré comme doté de valeur au premier sens du terme. En termes bancaires, une « valeur » est, typiquement, une lettre de change, une action ou une obligation, un billet de banque. Plus généralement, c'est ce qu'on appelle aussi un « bien ». Mais ce terme de « bien » tend à s'appliquer plutôt aux valeurs concrètes (par exemple on peut dire d'une montre, du moins si elle est « de prix », qu'elle constitue un « bien » ou une « valeur » dans un patrimoine), alors qu'il existe aussi des « valeurs » en un sens abstrait : par exemple, « la famille », « le travail », « la religion », « l'art », peuvent être considérés comme

\footnotetext{
8 « Grandeur » est le terme utilisé par L. Boltanski et L. Thévenot dans De la justification. Les économies de la grandeur (op. cit.) de façon à éviter l'incertitude sémantique associée au mot « valeur » (même si cet évitement n'a pu être réalisé dans la traduction anglaise de l'ouvrage, où c'est le terme « worth » qui a été choisi, équivalent à « valeur »). D'autres chercheurs ont également essayé de contourner l'obstacle : ainsi le philosophe Bruno Latour (in Enquête sur les modes d'existence, Paris, La Découverte, 20 I2, p. 434435), et à sa suite l'anthropologue Thierry Bonnot, proposent de remplacer « valeur » par « valence », terme emprunté à la fois à la chimie, où il renvoie au « nombre de liaisons qu'un atome engage avec d'autres atomes dans une combinaison », et à la psychologie, où il signifie la « puissance d'attraction ou de répulsion qu'exercent une activité ou un objet » (Th. Bonnot, L'Attachement aux choses, op. cit. p. 188). Plutôt que de chercher à contourner l'obstacle de la polysémie, nous resterons au plus près du vocabulaire utilisé par les acteurs, quitte à en différencier soigneusement les différentes significations.
} 
des « valeurs », autrement dit des entités auxquelles on accorde de l'importance, du prix. Le théoricien du discours Chaïm Perelman souligne ainsi « l'importance d'une distinction, trop souvent négligée, entre les valeurs abstraites, telles que la beauté ou la justice, et des valeurs concrètes, telles que la France ou l'Eglise. La valeur concrète est celle qui s'attache à un être particulier, à un objet, à un groupe ou à une institution, conçus dans leur unicité $)^{9}$. Les valeurs abstraites tendent à focaliser l'attention des enquêtes d'opinion, qui demanderont par exemple aux enquêtés: «Pour vous, la famille est-elle une valeur très importante, importante, peu importante ou pas importante du tout? 》, ou encore : "Parmi ces valeurs, ditesnous laquelle est la plus importante pour vous : la famille, le travail, l'amour... ? 》 - etc. Si donc ce deuxième sens du mot « valeur » est assez familier au monde économique lorsqu'il s'agit de valeurs concrètes, il l'est davantage au monde politique s'agissant de valeurs abstraites, que ce soit pour les citoyens ordinaires, les politiciens ou les politologues (on opposera ainsi les « valeurs de la droite » aux « valeurs de la gauche »). Nous appellerons ce deuxième sens du mot valeur la « valeur-objet $»$.

Enfin, la troisième signification du mot « valeur » renvoie non plus à une appréciation, comme la première, ni à un objet concret ou abstrait, comme la deuxième, mais au principe sous-tendant une évaluation : par exemple, dire « Ce film est très beau » implique que pour le locuteur en question, dans le contexte d'énonciation en question et à propos de l'objet en question, la « beauté » est une valeur. D'autres valeurs, au sens de principes d'évaluation, amèneront d'autres jugements : par exemple la valeur de moralité pour « Ce film est complètement indécent 》, la valeur d'efficacité pour « On y croit d'un bout à l'autre », la valeur de plaisir pour «C'est un vrai régal », la valeur de responsabilité pour « C'est un formidable message politique », la valeur d'authenticité pour " Ça manque de sincérité », ou encore la valeur économique pour « II va rapporter beaucoup d'argent ». Nous appellerons ce troisième sens du mot valeur la « valeur-principe ». La différence entre « la » valeur au premier sens (valeur-grandeur) et « une » valeur au troisième sens (valeurprincipe) est homologue à la différence entre « la » qualité d'un objet et « une » qualité de cet objet, autrement dit une propriété motivant sa valorisation ${ }^{10}$.

${ }^{9}$ Ch. Perelman, L'Empire rhétorique, op. cit. p. 48. Dans le même ordre d'idées, l'on a pu dénoncer l'inférence « subreptice » par laquelle une chose « qui a une valeur devient elle-même une valeur. On passe ainsi du verbe "avoir" au verbe "être", sans légitimation aucune. "X a une valeur" devient "X est une valeur" » (Cyril Arnaud, Axiologie 4.0. Proposition pour une nouvelle axiologie, wnw.axiologie.org, p. 15).

${ }^{10}$ Albert Ogien fait lui aussi la différence entre le deuxième et le troisième sens des « valeurs 》, mais en réduisant le premier aux seules valeurs abstraites, qu'il assimile à des institutions, sans y inclure les valeurs concrètes, autrement dit les biens : « Les valeurs renvoient tantôt à des institutions sociales (famille, nation, religion, laïcité, République), tantôt à des principes qui fondent ce qui est tenu pour notre commune humanité (dignité, liberté, égalité, honneur, responsabilité, fidélité, générosité). Cette différence est importante : alors que les institutions sont contingentes et font en permanence l'objet d'une critique qui les rend caduques ou les transforme, les principes possèdent une certaine permanence et sont les ressources invariables qui servent à élaborer cette critique » (A. Ogien, « Qu'est-ce qui fait valeur dans notre société ? », art. cit. p. 28). 
La « valeur-objet » (deuxième sens) et la « valeur-principe » (troisième sens) sont parfois très proches l'une de l'autre, dès lors que la première renvoie à un objet abstrait et non pas concret. Elles ne se différencient alors que parce que la valeurprincipe a pour propriété d'être une butée de l'argumentation : par exemple, dire " C'est bien parce que c'est de l'art » fait de l'art une valeur au troisième sens - un principe gouvernant une évaluation -, alors que dire " L'art est une valeur parce qu'il est porteur de beauté ») en fait une valeur au deuxième sens, c'est-à-dire un bien, objet d'une évaluation positive - la beauté étant alors la valeur au troisième sens, le principe de cette évaluation. C'est ce qu'on nomme le caractère autotélique de la valeur-principe, autrement dit le fait qu'elle est à elle-même son propre but, sa propre nécessité : « Une valeur se présente comme une fin lorsqu'elle met un terme à la série des "pourquoi" justificateurs de l'action », notait Célestin Bouglé "l. La valeur, prise en ce sens, est ce qui cause l'évaluation, alors que la valeur-grandeur et la valeur-objet résultent de l'évaluation : d'où l'intérêt majeur d'étudier ces « valeurs-principes », plus abstraites, peu familières au sens commun et peu analysées par les outils du monde savant; c'est à elles que sera consacrée la troisième partie de cet ouvrage.

(chapitre 6: « Les trois sens du mot "valeur"»)

\section{Objets d'attribution de valeur}

Les choses, les personnes, les actions, les états du monde : voilà les quatre catégories auxquelles s'appliquent les attachements, les mesures, les jugements qui attestent (aux yeux des acteurs) ou confèrent (aux yeux des sociologues) de la « valeur »au sens premier de grandeur, importance, qualitél2 . Une fois valorisées, les choses deviennent des biens (concrets), c'est-à-dire des « valeurs » au deuxième sens du terme ; les personnes deviennent des êtres de qualité, dotés de vertus telles que la gaîté, l'honnêteté, le courage ${ }^{13}$, ou de marqueurs d'excellence tels que les objets de luxe et le loisirl4, ou encore d'opérateurs de distinction tels que le « capital

\footnotetext{
" C. Bouglé, Leçons de sociologie sur l'évolution des valeurs, op. cit. Bouglé notait que le terme « autotélisme » appliqué aux valeurs provient du philosophe et psychologue américain James Mark Baldwin dans sa Théorie génétique de la réalité. Le Pancalisme (19|5), traduite chez Alcan en 1918.

${ }^{12}$ Nous utiliserons le mot « chose » lorsqu'il s'agit du sens concret, où les « choses » sont des êtres inanimés, et le mot « objet » lorsqu'il s'agit du sens abstrait et général (par exemple, l'« objet » d'une évaluation). Cette distinction objet/chose ne recoupe donc pas exactement celle proposée par le sociologue américain Fernando Dominguez Rubio, qui fait des « choses » des « processus matériels 》 et des objets les « positions » assignées à ces choses lorsqu'elles participent à différents régimes de valeur et de signification » ("On the discrepancy between objects and things. An ecological approach", Journal of material culture, 2016, p. 5).

${ }^{13}$ Notons que Rickert va même jusqu'à assimiler les « biens éthiques » aux personnes : « Les biens éthiques sont des personnes (...). Nous ne parlons d'éthique que dans la mesure où, en tant que philosophie "pratique", elle fait de l'homme agissant son objet » (H. Rickert, Le Système des valeurs, op. cit, p. 152).

${ }^{14}$ Cf.Thorstein Veblen, Théorie de la classe de loisir, 1899, Paris, Gallimard, 1970.
} 
économique », le « capital culturel » ou le « capital social $\rangle^{15}$; les actions (qu'il s'agisse d'un sourire, d'un acte de sauvetage ou de la participation à une corrida) deviennent des actions justes en tant que justifiées par leur conformité à des normes, voire des actes héroïques ; et les états du monde (du climat à l'état de paix ou au régime démocratique) deviennent eux aussi des biens, mais abstraits et non plus concrets.

S'il est important d'opérer ces distinctions entre les objets d'attribution de valeur, c'est qu'elles sont souvent ignorées ou sous-estimées ${ }^{16}$. Ainsi la science économique, en se concentrant sur le prix, tend à réduire implicitement la problématique de la valeur à la valeur des choses ${ }^{17}$, tandis que la philosophie morale, en se concentrant sur l'éthique, tend à la réduire à la valeur des personnes ${ }^{18}$, et que la sociologie, en se concentrant sur les normes, tend à la réduire à la valeur des actions. Aucune de ces focalisations n'est erronée, mais elles sont partielles : pour comprendre ce qu'est une compétence axiologique, il importe de prendre en compte l'ensemble des possibilités qu'elle est susceptible de mobiliser.

Concernant l'attribution de valeur aux personnes, il est intéressant de noter que c'est sur elle, plutôt que sur la chose mesurable et échangeable, que portaient en priorité les premières acceptions attestées du mot valor, notamment à travers la bravoure ${ }^{19}$.

${ }^{15}$ Cf. P. Bourdieu, La Distinction, op. cit.

${ }^{16}$ A l'inverse, certains dictionnaires distinguent les deux sens de « valeur » attribuée aux choses (tel le prix) et de « valeur » attribuée aux personnes (telle la bravoure), au point d'en faire des entrées lexicographiques différentes ( $c$. ..-Cl. Guerrini, « Les valeurs dans l'argumentation », op. cit. p. 36) - mais au prix, dès lors, d'une occultation de leurs points communs, ainsi que de leurs conditions d'articulation. L'évolution lexicographique va cependant dans le sens d'une unification des deux sens grâce à la prise en compte du jugement de valeur : «Après avoir longtemps fait coexister deux significations radicalement distinctes de la valeur selon le support qu'elle concernait (« prix ou valeur d'un bien » et « valeur, vaillance d'une personne »), sans égard pour la nature de l'évaluateur, les critères du jugement de valeur ont fini par être mentionnés pour eux-mêmes, ainsi que leur dépendance reconnue à l'égard du sujet évaluateur et des normes de la collectivité. C'est donc au processus d'explicitation des propriétés de l'évaluation et à la rupture avec son caractère d'évidence que l'évolution des dictionnaires nous donne accès » (ibid. p. 50).

${ }^{17}$ Célestin Bouglé avait déjà alerté sur ce problème de réduction de la notion de valeur à la valeur économique (cf. Leçons de sociologie sur l'évolution des valeurs, op. cit. p. I5).

${ }^{18}$ D'où la tension, notée également par Bouglé, entre un pôle « réaliste » et un pôle « idéaliste » de la valeur: « Le monde des valeurs a deux pôles. Le sentiment de la valeur a deux origines. La valeur c'est la vertu de l'homme capable de tous les sacrifices. Et, d'un autre côté, la valeur c'est le prix des choses au marché. Réalisme économique ici, et là, idéalisme moral. La distance paraît, au premier abord, difficile à franchir » (ibid. p. 63).

${ }^{19}$ « Le Dictionnaire historique de la langue française nous enseigne que ce terme apparaît en français en 1080, du latin classique valor, valoris. Dans la Chanson de Roland, un double sémantisme est attesté dès son premier emploi. En effet, le mot signifie à la fois « qu'une personne est estimée pour son mérite, ses qualités » et s'emploie aussi à propos de « la qualité, de l'intérêt d'une chose », d'où avoir valeur. Par extension, la première acception du terme renvoie à la notion d'importance et de bravoure. Ce n'est qu'à partir du XIII ${ }^{\circ}$ siècle que le terme désigne « le caractère mesurable d'une chose, d'un bien, en tant qu'il est susceptible d'être échangé » (Gilda Bouchat, « Le soubassement théologique de l'art dans les discours esthétiques au XVIII ${ }^{\circ}$ siècle », in Danièle Lories (éd.), L'Art en valeur, Paris, L'Harmattan, 20 I I, p. 105). Pour une analyse détaillée des différents traitements du mot « valeur » dans les dictionnaires, cf. J.-Cl. Guerrini, « Les valeurs dans l'argumentation », op. cit. p. 30 et passim. 
Remarquons également qu'il existe des êtres au statut constitutivement ambigu entre chose et personne : c'est le cas des « objets-personnes » que sont les reliques, les fétiches et les œuvres d'art20 ; c'est le cas surtout des animaux, qui peuvent passer de l'un à l'autre statut selon qu'ils sont traités comme des biens utilitaires (quasi-choses) ou comme des animaux de compagnie (quasi-personnes). D'où la récurrence et la virulence des débats sur le traitement qu'il convient de leur accorder - au nombre desquels figurent, nous le verrons, les débats sur la corrida. Nous observons là l'importance de la dimension contextuelle, sans laquelle il n'est pas de sociologie qui vaille, et que la question des valeurs rend particulièrement incontournable.

Tandis que la science économique se concentrait sur la valeur des choses, des avancées importantes dans l'histoire des sciences humaines et sociales ont été accomplies lorsque la problématique de la valeur s'est étendue aux autres catégories d'objets : les états du monde, avec les enquêtes sociologiques quantifiées sur les valeurs (nous le verrons au prochain chapitre) ; les personnes, comme y incitent notamment les travaux de l'anthropologue Arjun Appadurai ${ }^{2 !}$, qui « postule que la valeur des choses doit être analysée dans son rapport à la valeur des personnes (...), investies de valeurs différentes et placées sur le même plan que la valeur des choses \22 $^{22}$; et les actions, dont la conformité aux valeurs est réglée par des normes. Les actions ont été étudiées dans une perspective axiologique par les sociologues Luc Boltanski et Laurent Thévenot dans leur ouvrage sur la « justification » des actions, c'est-àdire sur la validité qui leur est accordée en fonction des différentes catégories de normes - la justification constituant ainsi un sous-ensemble de l'évaluation lorsque celle-ci est appliquée aux actions. L'anthropologue David Graeber proposera lui aussi de « créer une théorie de la valeur basée sur la supposition que ce qui est, en dernier analyse, évalué, ce ne sont pas des choses mais des actions $\gg^{23}$, sans paraître toutefois envisager qu'il n'y ait pas à choisir entre les deux, les acteurs pouvant évaluer aussi bien des choses que des actions, selon des modalités certes différentes.

(chapitre $7:$ : Valeur-grandeur : ce que valent les choses »)

\section{Reconnaître une valeur-principe}

Les valeurs sont multiples, même si leur nombre n'est pas infini : à chaque objet évalué peut s'appliquer non pas une seule mais plusieurs valeurs, selon les propriétés de l'objet en question, les dispositions du sujet évaluateur et les caractéristiques du

\footnotetext{
${ }^{20}$ Cf. N. Heinich, « Les objets-personnes. Fétiches, reliques et œuvres d'art », Sociologie de l'art, n 6, 1993.

${ }^{21}$ Cf. A. Appadurai, The Social Life of Things. Commodities in Cultural Perspective, Cambridge University Press, 1988.

22 Jean-Pierre Warnier, « Les politiques de la valeur », Sociétés politiques comparées. Revue européenne d'analyse des sociétés politiques, $n^{\circ} 4$, avril 2008.

${ }^{23}$ D. Graeber, The False Coin of our Dreams. Toward an Anthropological Theory of Value, New York, Palgrave, 2001, p. 49.
} 
contexte d'évaluation. Contrairement à « la » valeur (au premier sens), qui supporte mal de n'être pas unique et suscite inévitablement, dans ce cas, des conflits quant à la « juste » valeur, « les » valeurs (au troisième sens) cohabitent sans mal au sein d'un même processus d'évaluation : un plat au restaurant peut sans problème être jugé à la fois « bon », « beau », « sain » et « bon marché », ou bien encore « beau mais pas très bon », « excellent mais trop gras », « bon et beau mais trop cher » - et c'est la détermination de sa « valeur », au sens premier de « grandeur 》, qui se complexifiera alors. Exclusives les unes des autres, les valeurs n'en sont pas pour autant indissociables, pouvant se combiner entre elles ou se renforcer - telles l'ancienneté et l'authenticité, ou bien la fonctionnalité et la modernité. Elles peuvent d'ailleurs aussi, tout autant, se contredire, telles l'individualité et la solidarité. D'où la complexité du travail d'évaluation.

Cette pluralité des valeurs est constitutive de tout acte évaluatif, quoiqu'en pensent les tenants d'une vision uniciste du monde ${ }^{24}$. Comme le soulignait Raymond Polin, « l'évaluation est une hiérarchie en acte », qui « prend la forme d'un choix entre deux termes. Une valeur n'est jamais inventée seule, l'invention réside dans la préférence, le choix créateur des termes mis en balance. On pense une valeur par opposition à une autre $\gg^{25}$ : il existe bien, autrement dit, un système des valeurs, pluriel, hiérarchisé, oppositionnel, donc interdépendant. Et cette pluralité ne se limite pas à la triade du beau, du bien et du vrai chère au « rationalisme philosophique »: « Le pluralisme atomistique des valeurs rend cette unité mathématiquement improbable. C'est la plus grande dispersion qui est la plus vraisemblable $\gg^{26}$. Contrairement à ce que postule spontanément le réductionnisme logiciste, le pluralisme est toujours plus adapté aux affaires humaines que le monisme : cela vaut pour les valeurs comme pour la causalité.

Mais à quoi, concrètement, reconnaît-on une valeur?

II existe à cette question une réponse purement sémantique, mais qui n'est qu'indicative : beaucoup de valeurs ont un suffixe en « té », comme « liberté », 《 égalité 》, 《 fraternité 》 « beauté 》, 《 authenticité », « propreté 》, « fonctionnalité 》, 《légalité », etc ${ }^{27}$. Mais la règle n'est pas absolue : la « décence », le « travail », le « plaisir », le « savoir », la « force », l'« élégance », peuvent aussi fonctionner dans

\footnotetext{
${ }^{24}$ Bouglé remarquait ainsi que «Tout le monde ne prend pas si aisément son parti de cette incoordination. II y a des esprits qu'elle fait souffrir et qui veulent à tout prix retrouver, jusque dans la diversité des tendances, le principe d'unité sans lequel l'intégrité même de leur personne leur paraîtrait menacée » (Leçons de sociologie sur l'évolution des valeurs, op. cit. p. 46). Lui-même donnait spontanément comme exemples les valeurs « scientifique ou économique, esthétique ou morale » (ibid. p. 50), ou encore « économique ou esthétique, religieuse ou morale » (ibid. p. 52).

${ }^{25}$ R. Polin, La Création des valeurs, op. cit. p. 102.

26 Ibid. p. 228.

${ }^{27}$ «Sans qu'aucune règle de suffixation puisse être tenue pour convaincante, on ne peut tenir pour négligeable la fréquence des mots en -té dans notre liste (61\% des dés-adjectivaux, 24\% du total), qui témoigne de la forte tendance des mots-valeurs à être dérivés de bases adjectivale » (J.-Cl. Guerrini, « Les valeurs dans l'argumentation », op. cit. p. 265).
} 
le discours comme des valeurs. Force est donc de faire appel à des critères moins formels et plus discriminants, mais aussi plus complexes. Procédons par la négative, en considérant ce qu'une valeur n'est pas.

Tout d'abord donc, une valeur n'est ni une norme, ni une règle, ni une loi : celles-ci sont des applications de valeurs, lesquelles en justifient la création. Une première conséquence de cette spécificité est que, pour qu'une valeur puisse fonctionner comme telle, il faut qu'elle puisse être comprise et utilisable par tous : autant on peut ne pas connaître l'existence d'une loi ou d'un règlement, autant tout adulte engagé dans la vie sociale sait ce que c'est que l'« honnêteté », la « solidarité » ou la « bonté ». Une valeur est à large spectre, temporel autant que spatial : elle implique à la fois le long terme et l'universalité, en tout cas présumée. Autrement dit, elle ne joue son rôle de valeur qu'à condition d'être à peu près commune à tous les participants d'une même culture. De ce fait, elle n'est pas accessible à la modification volontaire ou à court terme : contrairement à une norme et, plus encore, à une règle ou à une loi, l'on ne peut décider que telle valeur est obsolète ou, au contraire, valide : tout au plus peut-on inciter à son abandon ou à son adoption. C'est pourquoi les changements de valeurs résultent de processus longs, diffus et collectifs. Par exemple, en ce qui concerne la valeur de décence dans le monde occidental, les lois et, surtout, les normes d'exposition du corps dans l'espace public se sont considérablement assouplies en deux ou trois générations, et l'importance accordée à cette valeur a probablement diminué, mais la décence n'en demeure pas moins une valeur, même faible.

En outre une valeur - au sens de principe d'évaluation et de justification - n'est, nous l'avons vu, ni « la » valeur (grandeur) ni un bien, un objet « de valeur » : en tant que facteur de valorisation, une valeur est l'origine de l'évaluation, et non pas son résultat, comme pour «la » valeur et pour les biens. La chose se complique toutefois du fait que des transferts sont possibles du bien au principe, ou du principe au bien, selon la forme du jugement : le même terme peut se retrouver dans l'une ou l'autre position énonciative. Par exemple, dans « C'est formidable, il a enfin un travail ! », le travail est un bien, objet d'une évaluation positive (deuxième sens) ; en revanche, dans « II est bien ce tableau, au moins il y a du travail ! », le travail est une valeur, un principe (troisième sens) permettant d'évaluer positivement l'objet en question, donc de lui conférer de « la » valeur (premier sens), faisant de lui « une » valeur au sens de bien (deuxième sens).

Mais, dans ce dernier exemple, le mot « travail » ne fonctionne-t-il pas comme un simple critère plutôt que comme une valeur? Le travail ne serait-il pas, dans l'esprit de l'auteur de cette assertion, un critère parmi d'autres permettant d'établir dans quelle mesure un objet possède, ou pas, cette « valeur »- au sens de principe d'évaluation - qu'est la beauté ? Nous verrons plus en détail, au prochain chapitre, ce qui distingue un critère d'une valeur, et pourquoi une telle distinction importe ; mais nous pouvons dès à présent poser ceci : à la différence d'un critère factuel tel que, par exemple, le temps mis à réaliser un objet, sa patine, ou sa date, une valeur est à elle-même sa propre fin. Ainsi, la valeur de ce tableau réside dans le 
travail qu'il a exigé, et ce travail n'a pas à être valorisé par autre chose que luimême. Ainsi encore, une fois qu'on a établi que ce grille-pain vintage fonctionne parfaitement, il n'y a pas à pousser plus loin l'argumentation pour peu que la valeur de fonctionnalité soit pertinente dans la situation : « II marche : je le prends ! »; idem pour l'ancienneté (« C'est un modèle des années cinquante : n'hésite pas! »), pour la rareté (« II n'en existe plus beaucoup des comme ça : vous n'en retrouverez pas un autre de sitôt! »), pour l'authenticité (« II a sa marque de fabrique : tu peux y aller ! ») - etc. Si le critère peut toujours se discuter (la marque de fabrique n'est pas forcément celle d'origine, la patine peut être fausse, la date peut être erronée), en revanche le principe qui fait de ce critère un motif de valorisation, parce qu'il en rend possible la mise en œuvre, ne s'autorise que de lui-même : pour peu que j'apprécie la fonctionnalité, ou l'ancienneté, ou l'authenticité, personne ne peut me convaincre que je me trompe.

Bref, une valeur ne repose sur rien d'autre qu'elle-même, est à elle-même sa propre fin (on l'appelle d'ailleurs parfois une « fin », remarque Putnam ${ }^{28}$ ). Elle est une butée de l'argumentation : c'est ce qu'on appelle l'autotélisme des valeurs. Célestin Bouglé donnait à ce propos l'exemple de la beauté : «Par un tableau, une statue, un drame, une symphonie, notre âme est plus facilement absorbée. Envahie par l'émotion esthétique, elle refuse de se demander à quoi sert la beauté, aussi bien qu'elle refuse de se demander ce que la beauté démontre. La beauté est le type de la valeur qui se suffit à elle-même $»^{29}$. Cet exemple n'était d'ailleurs pas pris au hasard : la beauté est une valeur essentielle dans le monde de l'art, or celui-ci est « le créateur des valeurs idéales par excellence. Car c'est dans ses œuvres, semble-t-il, que l'on voit le plus clairement la valeur se poser comme fin en soi $»^{30}$, notamment avec « l'art pour l'art » qui a remplacé les « fins religieuses $»^{31}$; aussi « le maximum d'intérêt désintéressé, c'est le propre de l'attitude esthétique $»^{32}$, ce désintéressement faisant de «la vie esthétique comme une introduction à la vie morale » : « L'art, même et surtout une fois son autonomie conquise, se trouve capable de seconder la moralité de plus d'une façon. II ne peut fleurir, disions-nous, que là où il y a désintéressement préalable de l'individu »-ce en quoi il constitue « un antidote contre les effets démoralisateurs que tend à produire l'empire exclusif de certaines autres valeurs, par exemple celui des valeurs économiques $\gg{ }^{33}$.

Etre une butée de l'argumentation : cette propriété des valeurs, quelles qu'elles soient, a déjà été repérée, notamment par l'économiste indien Amartya Sen, qui parle à son propos du caractère « élémentaire » des jugements de valeur : « Un jugement de valeur peut être appelé "élémentaire" pour une personne si

\footnotetext{
${ }^{28}$ Cf. H. Putnam, Faits/Valeurs : la fin d'un dogme, op. cit. p. I I.

${ }^{29}$ C. Bouglé, Leçons de sociologie sur l'évolution des valeurs, op. cit. p. 138.

$30 \mathrm{lbid}$.

${ }^{31}$ Ibid. p. 56.

32 Ibid. p. 138.

33 Ibid. p. 147.
} 
aucune révision concevable des suppositions factuelles ne peut l'inciter à réviser ce jugement. Si de telles révisions peuvent être effectuées, dans son système de valeurs, le jugement est "non élémentaire" $\gg{ }^{34}$. Autant dire que l'axiologie ne relève pas de la démonstration mais de la conviction, pas de la vérité mais de l'adhésion. Tout au plus pourra-t-on tenter de me persuader que j'ai tort de m'attacher à cette valeur à propos de cet objet (parce que la fonctionnalité vaut pour le design neuf mais pas pour l'ancien, ou que l'ancienneté n'est pas adaptée aux objets utilitaires, ou que l'affectivité n'est bonne que pour les midinettes, ou que la rareté est un truc de snobs), mais l'on ne pourra pas nier que c'est bien cette valeur-là qui me fait apprécier l'objet, pour le meilleur ou pour le pire - ni m'empêcher de le faire. En d'autres termes, toute valeur est autotélique. Et c'est principalement à cela qu'on la reconnaît.

Voilà qui invalide les tentatives pour fonder les valeurs soit par le conséquentialisme, ou justification par les conséquences (que se passerait-il si l'on contrevenait à telle valeur ?), soit par l'utilitarisme, ou justification par l'utilité (l'on adopterait telle valeur parce qu'elle nous serait utile $)^{35}$ - l'utilitarisme n'étant d'ailleurs qu'un conséquentialisme à échelle individuelle, supputant l'intérêt que le sujet pourrait tirer de l'application d'une valeur. En effet, le calcul rationnel des conséquences est sans effet sur la valeur : même si l'accueil d'un grand nombre de réfugiés doit entraîner des effets négatifs sur le pays hôte, la solidarité n'en reste pas moins une valeur. La seule façon d'invalider une valeur consiste à lui préférer, contextuellement, une autre valeur : par exemple, placer la sécurité, ou le confort, avant la solidarité. Mais c'est la mise en œuvre d'une valeur qui est discutable - pas la valeur elle-même.

Enfin, l'autotélisme invalide toute tentative pour justifier une valeur par une instance transcendante ou, au contraire, pour l'invalider par sa relativité. En appeler à la loi divine ou à l'identité nationale ne relève que de l'argument d'autorité dogmatique ( «Dieu veut que... », « I| est dans notre ADN de... »), mais pas de la déduction rationnelle. Inversement, qu'une valeur ne soit pas universelle (« Ça n'est pas une valeur pour tout le monde... ») n'enlève rien au fait que ceux qui y adhèrent la considèrent comme une valeur. Contrairement à toutes les tentatives pour rationaliser le rapport aux valeurs, l'adhésion à une valeur est sans justification ni appel : « II faut, parce que c'est comme ça ». Cela ne signifie bien sûr pas que les valeurs ne seraient pas argumentables, comme en témoignent les innombrables discussions sur l'opportunité d'attribuer telle valeur à tel objet; cela signifie que, en tant que principes de valorisation, elles sont considérées par le locuteur comme indiscutables, non négociables : «Vous n'allez quand même pas nier que la liberté est une valeur! »

\footnotetext{
${ }^{34}$ A. Sen, «The Nature and Classes of Prescriptive Judgments », Philosophical Quaterly, 17, n 66, 1967, p. 50.

${ }^{35}$ Sur le conséquentialisme et l'utilitarisme en éthique, $c f$. Th. Nagel, « Egalité » in Questions mortelles, 1979, Paris, PUF, 1983.
} 
Voilà un sérieux obstacle au réductionnisme rationaliste qui, en dépit des efforts déployés par certains, ne tient pas devant la nature assertive, non démonstrative et autotélique des valeurs : « Les valeurs ne se démontrent pas : elles se posent et elles s'imposent. Elles s'imposent, précisément parce qu'elles sont des valeurs $\rangle^{36}$.

(chapitre 9 : «Valeurs-principes : au nom de quoi l'on valorise »)

\section{De la valeur à l'acte d'évaluer : le tournant pragmatiste}

Plusieurs conclusions peuvent être tirées de ces études de cas où des experts évaluent les objets qui leur sont soumis. La première conclusion est qu'ils n'ont pas eux-mêmes entièrement accès à la logique axiologique de leurs propres actes : ils ne pourraient pas expliciter spontanément tous les principes d'évaluation qui ont été mis au jour, soit parce qu'ils n'en ont pas conscience, ou pas complètement, soit parce qu'ils ne les considèrent pas comme intéressants ou comme légitimes. C'est dire que, pour rendre compte du travail d'expertise, la réflexivité des acteurs ne suffit pas. Nous le verrons plus loin avec la critique des méthodes quantitatives en sociologie des valeurs, et nous venons de le vérifier sur pièces : même la méthode qualitative, par entretiens, ne produit que des résultats assez pauvres par rapport à la méthode par observation de situations, propre à l'approche pragmatique.

La deuxième conclusion est que, pour étudier les jugements de valeur, il n'est pas indispensable de viser l'explication des positions des acteurs par leurs propriétés : cette perspective explicative peut avoir, certes, sa pertinence, mais est-elle vraiment intéressante ? A-t-on réellement besoin d'une observation rapprochée pour deviner que, dans la commission municipale, le critique parisien âgé va écraser de son autorité les femmes provinciales plus jeunes, ou que l'avis scientifiquement élaboré du conservateur de l'Inventaire aura plus de poids que la moue de dégoût du responsable associatif ? Beaucoup plus éclairante en revanche est l'approche compréhensive, qui explicite les ressources axiologiques disponibles aux acteurs, les contraintes de la situation et les prises offertes par les objets soumis à évaluation. C'est un tout autre « style » de sociologie qui s'avère ici heuristique, en rupture avec la doxa héritée de trois générations d'enquêtes statistiques et de deux générations de paradigme bourdieusien.

La troisième conclusion est que la compréhension de ces actes évaluatifs exige de prendre en compte ces trois paramètres que sont les ressources des sujets évaluateurs (leur équipement axiologique), les propriétés des objets évalués (les prises qu'ils offrent à l'observation), et les caractéristiques de la situation d'évaluation (les contraintes normatives propres à l'épreuve de la décision). Sujet, objet, contexte : c'est bien dans la relation entre ces trois opérateurs que se produit la valeur, à l'issue de l'ensemble des opérations par lesquelles une qualité est affectée à un objet.

${ }^{36}$ J. Coenen-Huther, « Eugène Dupréel, philosophe, sociologue et moraliste », art cit. 
S'ensuit le retournement phénoménologique, qui fait de la valeur non pas un donné mais une création. Le phénoménologue Raymond Polin l'affirmait déjà : « Le propre de la valeur, c'est donc de n'être jamais un donné, mais d'être à chaque instant réinventée, continûment recréée, ou mieux, d'être invention et création en devenir, transcendance consciente en train de se réfléchir et de s'exercer $\rangle^{37}$.Toutefois, contrairement à la vulgate véhiculée par la phénoménologie, à la fois solipsiste (chacun pour soi) et individualiste (pas d'institutions, pas de contrainte externe, pas de processus de stabilisation), les valeurs ne sont pas créées ex nihilo, mais reconfigurées et mises à l'épreuve à partir de corpus existants ; et elles ne sont pas le produit du libre jeu des individus, mais sont fortement contraintes par des institutions, des régulations, des cadres cognitifs, juridiques, administratifs, relationnels, etc. C'est dire qu'elles ne relèvent pas que de la psychologie, contrairement à ce que suggérait le philosophe allemand Heinrich Rickert lorsqu'il proposait, à juste titre, de soumettre l'ontologie de la valeur à l'acte d'évaluer ${ }^{38}$.

C'est donc à condition de réintroduire dans l'analyse les dimensions du collectif, de l'institué, de la structure, que cette nécessaire remontée de la valeur à l'acte d'évaluation ${ }^{39}$ peut échapper à l'illusion, si bien dénoncée par Elias, de l'homo clausus - l'homme clos sur lui-même, défini indépendamment de toute relation, de toute détermination exogène. II devient alors possible d'élaborer une sociologie des valeurs qui rende compte de la complexité des évaluations produites par les acteurs en étant, comme nous venons de le voir, à la fois neutre, descriptive, empirique, pragmatique, et compréhensive.

(chapitre 4 : « Sociologie de l'évaluation : comment les experts évaluent »)

\section{Du tournant pluraliste au tournant grammatical}

La mise en évidence des contraintes et des régularités propres à chacun de ces systèmes pluriels de valeurs amène Boltanski et Thévenot à inscrire leur travail dans ce qu'ils nomment eux-mêmes une « grammaire » de la justification des actions par les acteurs, explicitant les régularités sous-jacentes, non dites mais partagées et (plus ou moins) connues de tous, grâce auxquelles les acteurs peuvent ressentir une action comme « juste ». C'est dire que la visée du sociologue n'est plus dès lors

\footnotetext{
${ }^{37}$ R. Polin, La Création des valeurs, op. cit. p. 72.

38 «Cependant, la valeur est liée en outre à un sujet qui évalue des objets, et on peut être alors d'avis qu'une réalité effective ne devient un bien - qu'un tableau devient une œuvre d'art - que par le fait qu'un sujet lui adjoint une valeur. (...) De même qu'il n'y a de plaisir que dans la mesure où il est ressenti, il n'y aurait de valeur que dans la mesure où des sujets les évalueraient. La valeur elle-même serait une réalité effective, plus exactement un être psychique, et une science des valeurs serait par conséquent une partie de la psychologie » (H. Rickert, Le Système des valeurs, op. cit. p. 63-64).

${ }^{39}$ «En revenant pour ainsi dire en arrière, nous devons tenter à présent de revenir de ce qui est devenu au devenir, des biens achevés à l'acte d'évaluation qui adjoint une valeur à la réalité effective et en fait par conséquent un bien » (ibid. p. 77). Cette remontée « des valeurs à l'évaluation » est également préconisée par les directeurs d'un ouvrage collectif dont la postface est justement intitulée "From values to valuation" (Tobias Brosch, David Sander (eds), Handbook of Value, Oxford, Oxford University Press, 2016).
} 
l'explication des conduites ou des représentations par la position des acteurs dans l'espace social, mais leur compréhension par la mise au jour des ressources et des contraintes dont ils disposent. En s'intéressant davantage à ce que les acteurs ont en commun (une grammaire) qu'à ce qui les différencie (des positions sociales), et en visant davantage l'explicitation de leurs systèmes de représentation que l'explication de leurs comportements effectifs par des paramètres externes, cette sociologie opère une profonde rupture avec le projet explicatif et, fondamentalement, critique, qui est devenu avec Bourdieu le paradigme dominant en sociologie $\mathrm{e}^{40}$.

Ce projet grammatical sera poursuivi dans le domaine axiologique par Boltanski lorsqu'il s'intéressera aux « compétences » évaluatives des acteurs s'inscrivant non plus dans une visée de « justice », comme avec la justification, mais dans une visée d'« amour $»^{41} ;$ ou bien à la question de l'empathie et, plus généralement, de la conduite à adopter face à la souffrance d'autrui ${ }^{42}$; ou encore à l'avortement et aux valeurs soustendant les controverses conduites à son sujet ${ }^{43}$. C'est de ce même projet grammatical que relèvent également un certain nombre de travaux menés dans la continuité de cette école de « sociologie pragmatique », appuyée sur une analyse compréhensive des « épreuves » évaluatives rencontrées par les acteurs, ou des « affaires » publiques. Citons par exemple celui de Cyril Lemieux sur la déontologie journalistique ${ }^{44}$, analysée selon un modèle de l'action mettant en œuvre trois « grammaires » : la « grammaire publique », ou de la distanciation, qui prescrit d'agir en s'appuyant sur des représentations collectives permettant le jugement par un tiers (ainsi du recueil des preuves avant d'écrire) ; la « grammaire naturelle », ou de l'engagement, qui prescrit l'action adossée à des « attractions » non justifiables devant un tiers (ainsi de l'échange de services, des amitiés...) ; et enfin la « grammaire de la réalisation », ou principe de réalisme. C'est de ce même projet que relèvent également mes propres enquêtes sur les controverses à propos de l'art contemporain - nous y reviendrons.

Le paradoxe toutefois de ces travaux issus de la sociologie pragmatique, c'est que le terme même de « valeurs » n'y apparaît pas ou fort peu (on lui préfère parfois celui de « grandeur », comme dans le sous-titre de De la justification), cette problématique n'étant abordée qu'indirectement alors même qu'il y est constamment question des principes axiologiques guidant les évaluations des acteurs. L'on peut comprendre cette prudence par le souci d'éviter la confusion avec la « sociologie des valeurs » par enquêtes statistiques, dont nous avons vu la prégnance en même temps que les limites, autant qu'avec la « sociologie morale » à visée normative, dont nous avons vu également qu'elle n'est guère qu'une déclinaison de la philosophie morale, voire de la morale tout court. En outre, l'assimilation spontanée du mot « valeur » aux « valeurs morales » voire, aujourd'hui, aux valeurs défendues par les partis de

\footnotetext{
${ }^{40}$ Tout cela a été développé in N. Heinich, Pourquoi Bourdieu, op. cit.

${ }^{41}$ Cf. L. Boltanski, L'amour et la justice comme compétences, Paris, Métailié, 1990.

${ }^{42}$ Cf. L. Boltanski, La souffrance à distance. Morale humanitaire, médias et politique, Métailié, 1993.

${ }^{43}$ Cf. L. Boltanski, La Condition foetale. Une sociologie de l'engendrement et de l'avortement, Paris, Gallimard, 2004.

${ }^{44}$ Cf. C. Lemieux, Mauvaise presse. Une sociologie compréhensive du travail journalistique et de ses critiques, Paris, Métailié, 2000.
} 
droite (l'expression « défense des valeurs » renvoyant immédiatement aux valeurs traditionnelles, familiales et religieuses) ne facilite guère, dans un monde universitaire souvent marqué à gauche, la prise au sérieux de la problématique axiologique comme objet d'investigation sociologique assumé comme tel.

Cependant, si le triple tournant - qualitatif, pluraliste, grammatical ou compréhensif dont nous venons de passer en revue quelques moments marquants fait véritablement rupture avec la « sociologie des valeurs » propre aux sondages d'opinion, c'est en tant que l'enquête s'y appuie empiriquement et sur l'observation de situations réelles, et sur des moments de controverses ou d'épreuves. C'est à cette double condition en effet que la pluralité des valeurs apparait dans sa dimension problématique, conflictuelle, plutôt que comme un catalogue de ressources axiologiques consensuelles, trop générales pour donner accès à la réalité des choix axiologiques opérés par les acteurs. Nous sommes là au cœur de ce qu'apporte la sociologie dite « pragmatique », née en France dans les années 1990 autour de Luc Boltanski et Laurent Thévenot, d'une part, et de Bruno Latour, d'autre part.

(chapitre $8:$ «Valeurs-objets : ce que les gens valorisent »)

\section{Structuralisme, interactionnisme, constructivisme}

L'analyse de ces quelques cas de dissonance axiologique nous a donc permis de vérifier que les représentations axiologiques - les « valeurs 》 aux trois sens du terme - dépendent à la fois des propriétés objectales (les caractéristiques de l'objet du jugement), des compétences axiologiques incorporées par les acteurs, et des contextes en lesquels se produit cette forme particulière d'interaction entre objets et sujets qu'est une évaluation (et il s'agit bien d'une interaction car les objets, grâce à leurs spécificités, peuvent faciliter ou entraver ce que l'on peut faire d'eux). Or cette triple dimension de l'expérience axiologique concerne aussi sa temporalité et, corrélativement, le statut des outils théoriques susceptibles d'en rendre compte.

En effet, les valeurs sont présentes trois fois dans le cours de l'expérience évaluative. Premièrement, elles existent avant la situation d'évaluation, en tant qu'appartenant à un répertoire de représentations partagées par les acteurs au sein d'une même culture, transmises par l'éducation, incorporées dans leur habitus. C'est là la dimension structurale, déterministe, de l'expérience commune, comparable à la grammaire que pratiquent les locuteurs d'une langue, ou à ces « cadres de l'expérience » mis en évidence par Erving Goffman, qui structurent et prédéterminent, sans qu'on en ait conscience, la façon dont nous percevons et traitons les moments de la réalité $v_{\text {vécue }}^{45}$ - exactement comme le font les « registres de valeurs ». De ce point de vue, la problématique des valeurs s'insère parfaitement dans le cadre d'une science sociale structuraliste.

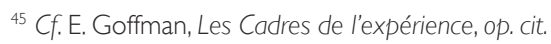


Deuxièmement, les valeurs s'élaborent aussi dans la situation d'évaluation, en s'exerçant concrètement sur tel ou tel objet, avec une efficacité variable, dont nous venons de voir qu'elle dépend pour beaucoup des rapports de force entre les différents porteurs des valeurs mobilisées. Là, nous avons affaire à la dimension à la fois pragmatique et interactionniste de l'enquête sociologique, attentive à la concrétude des actions et de leurs contextes, ainsi qu'à la grande variété de ressources dont disposent les acteurs pour négocier entre eux, notamment en situation de conflit. La tradition interactionniste en sociologie a ici toute sa place, même si elle se situe, théoriquement, à l'opposé de la tradition structuraliste ${ }^{46}$.

Troisièmement enfin, les valeurs se construisent après la situation d'évaluation, grâce à leur mise à l'épreuve au contact des objets et sous l'influence des contextes: elles peuvent ainsi se moduler, s'affiner, s'affirmer ou, au contraire, s'affaiblir ou se périmer, entraînant une réélaboration permanente du répertoire dont disposent les acteurs - exactement de même qu'avec l'usage d'une langue. C'est là la dimension constructiviste du programme sociologique, qui laisse toute sa place à l'évolution historique et aux capacités inventives des acteurs.

Autant dire qu'il n'y a pas à imposer a priori telle ou telle approche théorique : la pertinence des unes et des autres est avant tout fonction du moment de l'expérience auquel on s'attache. Plutôt donc que de se complaire dans de stériles luttes de clans entre partisans de tel ou tel programme théorique, mieux vaut assurer la prise en compte de ces trois moments - l'avant, le pendant, l'après de l'épreuve - ainsi que de ces trois dimensions - objectale, représentationnelle, contextuelle - en tant qu'elle constitue l'apport spécifique de la sociologie à la question des valeurs. En nous permettant de passer de l'essentialisme des valeurs à la contextualisation des opérations d'évaluation, elle ouvre un champ nouveau à la connaissance de la pluralité des modes de qualification et même, probablement aussi, de perception de l'expérience.

(chapitre 13: «Petits arrangements avec les valeurs »)

\section{En finir avec l'universalisme, en finir avec le relativisme}

S'abstenir de prétendre statuer sur l'origine ou la nature de la valeur ou des valeurs « en soi », c'est cultiver un parfait agnosticisme concernant ce que serait une supposée « essence » des valeurs. La seule certitude que nous ayons à leur égard est qu'elles sont, nous l'avons vu, des représentations, et plus précisément des représentations axiologiques, à la fois individuelles et collectives. Elles sont donc « culturelles » à ce

\footnotetext{
${ }^{46}$ Paradoxalement, c'est le grand sociologue interactionniste que fut Goffman qui a aussi produit, avec Frame Analysis, un chef-d'œuvre de sociologie structuraliste (cette contradiction a été analysée in N. Heinich, « Pour introduire à la cadre-analyse », Critique, n 535, décembre 1991, repris dans Comptes rendus à..., Paris, Les Impressions nouvelles, 2007).
} 
double titre : et en tant qu'elles sont des représentations mentales que se font les humains de ce qui est digne d'être apprécié ou loué, ce en quoi elles sont irréductibles à des faits de nature ; et en tant qu'elles sont des représentations collectives, ce en quoi elles sont irréductibles à des opinions individuelles, même si c'est à travers des expressions individuelles qu'elles se manifestent.

Faits de culture et non pas de nature, possédant une dimension collective et non pas seulement individuelle, les valeurs échappent ainsi à toute imputation d'universalité, du fait que les cultures sont elles-mêmes plurielles, dans le temps et dans l'espace. II est donc vain de partir en quête d'une universalité ou d'une objectivité des valeurs, de même que de leur origine ou de leur fondement : tout ce que l'enquête historique et sociologique peut mettre au jour, ce sont non pas des invariants, mais des phénomènes de moindre variation, autrement dit des valeurs plus constantes ou plus répandues que d'autres.

Un tel programme peut paraître désenchanteur à ceux qui attendent des sciences humaines et sociales qu'elles leur donnent accès à l'universalité des constituants de l'expérience humaine. Mais pour ceux qui s'intéressent aux modalités effectives de cette expérience plutôt qu'à ses fondements ultimes, la recherche des phénomènes de moindre variation est un programme plein de promesses excitantes - ne serait-ce que la promesse d'arracher la sociologie à la sphère des « humanités » pour la faire entrer de plein droit dans celle des « sciences sociales ».

Le problème est que la sociologie, pour éviter le piège de l'universalisme et de l'essentialisme, qui tendent à faire des valeurs des entités substantielles, objectives, intangibles ${ }^{47}$, a préféré, pour une large part, jeter le bébé avec l'eau du bain, en sacrifiant cette problématique sur l'autel du constructivisme, du relativisme et, plus généralement, de la critique du naturalisme - lequel n'a pourtant strictement rien à faire dans une expérience aussi entièrement et radicalement sociale que celle du rapport aux valeurs, comme le rappelait déjà Raymond Polin ${ }^{48}$.

Or, dans la mesure où la valeur n'est pas dans l'objet (un principe que Polin encore nommait « neutralité des œuvres $»^{49}$ ), mais dans un système de représentations partagées, contextuellement appliqué à un objet, l'objectivisme se marie fort bien avec la relativité des points de vue. C'est ce que signifiait déjà la célèbre parabole de Hume sur le goût du vin auquel, dans Don Quichotte, l'un trouve un léger goût de cuir tandis que l'autre lui trouve un léger goût de fer : il suffira de vider la barrique et de découvrir en son fond une clé et une lanière de cuir pour comprendre que l'un et l'autre avaient raison. Les goûts subjectifs, comme les jugements de valeur, s'enracinent dans des propriétés objectives, mais dont

\footnotetext{
47 Voir en annexe : « Humanités et sciences sociales à l'épreuve des valeurs 》.

${ }^{48}$ Cf. R. Polin, La Création des valeurs, op. cit. p. 141.

${ }^{49}$ «Une œuvre, dans la mesure où elle est réelle, est axiologiquement neutre, indifférente et indéterminante par rapport aux valeurs. (...) Par rapport à une œuvre donnée, toutes les évaluations sont possibles et toutes se valent. Ainsi pourrait-on énoncer le principe de la neutralité des œuvres » (ibid. p. 250).
} 
la perception est relative à l'observateur. C'est ce qu'affirmera à sa façon Yves Michaud dans son commentaire ${ }^{50}$, et dans sa conclusion : « Si l'on cherche des critères absolus et universels, qu'on se rassure : on n'en trouvera pas et ce n'est pas la peine de se désoler $\gg^{51}$.

Ainsi, en matière de valeurs, l'absence d'objectivité absolue ne signifie nullement la subjectivité : elle signifie la variabilité et des répertoires axiologiques disponibles, et des contextes, temporels et spatiaux. Cette variabilité n'empêche nullement l'accord sur des principes et des pratiques, la construction de collectifs, de routines et de traditions intellectuelles, la stabilisation de normes et de critères partagés, voire la création d'institutions - en d'autres termes ce qu'une certaine tradition philosophique aime appeler la « rationalité ». Simplement, cet accord est fondé sur la croyance des acteurs en des valeurs universelles, tout en se construisant par l'interaction effective entre des objets, des humains, et des contextes.

C'est pourquoi le constat de la relativité effective des valeurs n'implique nullement le relativisme, qui postule l'absence de toute objectivité, de toute universalité et, partant, de toute nécessité des jugements de valeur : le relativisme descriptif, qui se contente d'observer les variations, n'a rien à voir avec le relativisme normatif, qui en conclut à l'impossibilité de choisir entre les valeurs et, partant, de justifier qu'on y adhère ou qu'on veuille en imposer le respect ${ }^{52}$. Mais pour prendre conscience de cela, il faut avoir accepté la spécificité du regard sociologique, qui décrit sans prendre parti, y compris lorsqu'il décrit des prises de parti : spécificité dont on peut comprendre qu'elle demeure quelque peu étrangère au point de vue des hommes politiques et des citoyens ordinaires, mais dont il faut bien constater, malheureusement, qu'elle continue à être ignorée par maints sociologues.

C'est que, pour adopter la posture exclusivement descriptive et analytique du chercheur qui reconstitue un système sans pour autant prétendre à une visée normative, il faut renoncer à l'ambition de fonder une morale sur le savoir : la morale sert à agir de façon juste, tandis que le savoir sert à savoir (cet autotélisme signalant d'ailleurs que le savoir est, au moins dans notre culture occidentale, une valeur : ce pourquoi la production d'un savoir n'a pas à être justifiée par autre chose qu'elle-même). C'est dire que la sociologie des valeurs n'a rien à voir - on l'aura compris - avec une quelconque éthique.

(« Conclusion »)

\footnotetext{
${ }^{50}$ « II me semble tout à fait possible de maintenir à la fois une position relativiste et objectiviste, qui correspond assez bien à l'esprit de ce que Goodman qualifie de "relativisme sous contrainte de rigueur". Au sein d'une communauté d'évaluation qui est articulée à une communauté de production, les évaluations correspondent à la fois à des traits réels des objets et sont aussi relatives au jeu de langage en cause. Si bien que l'évaluation est relative aux règles et aux critères qui lui permettent d'exister et de s'exprimer » (Y. Michaud, Critères esthétiques et jugements de goût, op. cit. p. 24).

${ }^{51}$ Ibid. p. 102.

${ }^{52}$ Cette proposition a été développée in N. Heinich, Ce que l'art fait à la sociologie, op. cit.
} 


\section{Une solution sociologique}

Nous venons de voir se déployer le nœud de problèmes que suscite la question des valeurs : outre leur réduction aux intérêts ou aux normes, qui leur fait courir le risque d'une relative invisibilité, l'essentiel de ces problèmes tient à l'opposition récurrente entre deux conceptions : l'une, « réaliste », qui postule le caractère intrinsèque, factuel, objectif, nécessaire et rationnel des valeurs ; l'autre, « nominaliste » (au sens de la « querelle des universaux »), qui les considère comme des propriétés extrinsèques aux chose jugées, relevant des représentations humaines et - dans les versions les plus radicales - entachées de subjectivité, d'arbitraire voire d'irrationalité.

Le lecteur aura sans doute compris que, face à cette alternative, l'auteur de ces lignes a pris parti pour la seconde option, à condition du moins d'accepter un certain nombre de nuances : accepter donc que, premièrement, il existe des propriétés intrinsèques des choses qui donnent plus ou moins prise à telle ou telle évaluation ; accepter que, deuxièmement, les représentations axiologiques n'en sont pas moins des « faits sociaux », donc à traiter, selon le précepte de Durkheim, « comme des choses » à étudier ; accepter que, troisièmement, l'absence d'objectivité des valeurs ne signifie pas leur subjectivité, car entre les objets et les sujets s'interpose cette réalité éminemment sociale que sont les systèmes de représentations et les institutions qui les stabilisent et les rendent agissantes; accepter que, quatrièmement, l'absence de nécessité transcendantale (qu'elle soit naturelle ou divine) des valeurs n'implique en rien leur arbitraire, car les nécessités propres au fonctionnement des sociétés sont infiniment plus puissantes que les forces de la nature (sans parler de celles, peu « probables » au sens littéral du terme, des divinités); et accepter enfin que, cinquièmement, les impératifs de cohérence et d'argumentabilité inhérents à la pratique des valeurs suffisent largement à inscrire celles-ci dans la rationalité, pour peu qu'on ne réduise pas celle-ci à une « raison » intellectualiste et logiciste.

Bref, à l'égard des valeurs, c'est un « relativisme sous contrainte de rigueur » qui devrait s'imposer, c'est-à-dire un « relativisme descriptif $»^{53}$, qui constate et analyse les variations des systèmes axiologiques mais sans pour autant en conclure, comme le voudrait la doxa post-moderne, que « tout se vaut »- puisque l'on ne peut passer du « est » au « doit être », donc du « est relatif » au « doit être relativisé ».

(annexe : « Humanités et sciences sociales : l'épreuve des valeurs »)

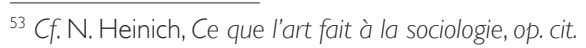

